\title{
Audiologic prognostic factors for hearing preservation following vestibular schwannoma surgery
}

\author{
Aleksandra Ochal-Choińska ${ }^{1, A-F}$, Magdalena Lachowska ${ }^{1, A, C-F}$, Katarzyna Kurczak ${ }^{2, D-F}$, Kazimierz Niemczyk ${ }^{1, E, F}$ \\ ${ }^{1}$ Department of Otolaryngology, Medical University of Warsaw, Poland \\ ${ }^{2}$ Center for Foreign Languages, Medical University of Warsaw, Poland \\ A - research concept and design; B - collection and/or assembly of data; C - data analysis and interpretation; \\ $D$ - writing the article; $E$ - critical revision of the article; $F$ - final approval of the article
}

\section{Address for correspondence}

Magdalena Lachowska

E-mail:mlachowska@wum.edu.pl

Funding sources

None declared

Conflict of interest

None declared

Received on July 26, 2017

Reviewed on November 19, 2017

Accepted on May 5, 2018

Published online on February 19, 2019

\section{Cite as}

Ochal-Choińska A, Lachowska M, Kurczak K, Niemczyk K.

Audiologic prognostic factors for hearing preservation

following vestibular schwannoma surgery. Adv Clin Exp Med.

2019;28(6):747-757. doi:10.17219/acem/90768

DOI

10.17219/acem/90768

\section{Copyright}

Copyright by Author(s)

This is an article distributed under the terms of the

Creative Commons Attribution Non-Commercial License

(http://creativecommons.org/licenses/by-nc-nd/4.0/)

\begin{abstract}
Background. Postoperative hearing loss after vestibular schwannoma (VS) removal still remains a lifelong problem for the patients. The present study analyzes the problem of hearing preservation after VS removal from a different angle than available professional literature on this topic.

Objectives. To identify audiologic factors which determine the extent of hearing loss in patients operated on for VS.

Material and methods. The study group included 86 patients operated on due to VS accessed via the middle cranial fossa. The analyses involved the effect on absolute hearing loss, which was calculated on the basis of the results of pure-tone audiometry performed pre-and postoperatively, and factors included in the preoperative audiologic tests, such as pure-tone audiometry, speech audiometry, auditory brainstem response (ABR), and impedance audiometry.
\end{abstract}

Results. The following parameters were demonstrated to have a prognostic value: 1. hearing thresholds at $125 \mathrm{~Hz}, 500 \mathrm{~Hz}$ and $1,000 \mathrm{~Hz}$ for the operated ear, Pure Tone Average (PTA) - calculated specifically at $500 \mathrm{~Hz}, 1,000 \mathrm{~Hz}$ and $2,000 \mathrm{~Hz}$ and at $500 \mathrm{~Hz}, 1,000 \mathrm{~Hz}, 2,000 \mathrm{~Hz}$, and 4,000 Hz for the operated ear, and normal audiometric curve; 2. speech discrimination ranging from $55 \mathrm{~dB}$ to $75 \mathrm{~dB}$ for the operated ear, speech detection threshold (SDT) in the operated ear and interaural difference at 25-35 dB (non-operated vs operated ear); 3 . presence of wave $V$, the values of $I-V$ and $I I I-V$ intervals for the operated ear, the amplitude of wave $V$, and the interaural ratio of wave $V$ amplitudes; 4 . intensity level for obtaining stapedial reflex or an abnormal reflex at Ipsi $500 \mathrm{~Hz}, 1,000 \mathrm{~Hz}$ and 2,000 Hz, and Contra $500 \mathrm{~Hz}, 1,000 \mathrm{~Hz}, 2,000 \mathrm{~Hz}$, and 4,000 Hz.

Conclusions. The better the preoperative hearing status, the more substantial surgery-related hearing loss was observed. A number of preoperative parameters of the basic diagnostic set of audiologic tests present a prognostic value for the degree of surgery-related hearing loss in VS patients.

Key words: hearing loss, neuroma, middle cranial fossa, audiometry, auditory evoked potentials 


\section{Background}

The development in the field of medicine has facilitated the shift of the primary aim of vestibular schwannoma (VS) removal surgery from tumor removal to the smallest possible impairment of neurological functions. The impairment of facial nerve function is mostly transient. However, postoperative hearing impairment still remains a lifelong problem for the patients.

It is therefore necessary to determine the factors responsible for the extent of hearing loss resultant from the surgery and to employ them in the optimization of the therapeutic process. A review of the professional literature revealed a paucity of detailed and multidimensional analyses of possible prognostic factors included in the basic panel of audiologic diagnostic tests for VS patients which would determine the extent of surgery-related hearing loss.

\section{Objectives}

The main aim of the study was to identify audiologic factors which determine the extent of hearing loss in patients operated on for VS. The analysis included the following audiologic tests: pure-tone audiometry, speech audiometry, auditory brainstem response (ABR), and impedance audiometry with particular attention paid to stapedial reflex.

Some authors have attempted to investigate the role of pure-tone audiometry or speech audiometry outcomes as prognostic factors. However, studies describing the predictive value of ABR are scarce. The available analyses have concentrated on few parameters of response recording, neglecting such data as the values of amplitudes of individual waves.

\section{Material and methods}

This retrospective analysis covered data obtained from audiologic tests and case histories of patients operated on for VS via middle cranial fossa approach which is a technique facilitating hearing preservation. The study involved 86 patients with a postoperative histopathological confirmation of VS.

All the surgical procedures were performed by the same experienced otosurgeon with substantial expertise in skull base surgery. The surgeries of the patients analyzed were carried out within 6 years, however, the potential influence of his gradually growing experience and on surgery-related hearing loss was ruled out ( $\mathrm{p}>0.05)$.

Each study group patient underwent diagnostic and medical procedures according to a standard protocol implemented by Department of Otolaryngology, Medical University of Warsaw (Poland) concerning patients with a suspected tumor of the cerebellopontine angle. According to the protocol, each patient underwent audiologic tests, including pure-tone audiometry, speech audiometry, impedance audiometry with stapedial reflex testing, and ABR no later than a month prior to the procedure. Magnetic resonance imaging (MRI) was used to confirm the presence of a tumor in the cerebellopontine angle region in each patient.

The follow-up tests included pure-tone audiometry alone or pure-tone audiometry and speech audiometry performed 3 months after the surgery.

This study presents the analysis of a number of factors (157 individual parameters) included in the parameters of the audiologic tests, imaging test data and case histories in terms of their possible influence on surgery-related hearing loss.

The following parameters were assessed with pure-tone audiometry: the values of hearing thresholds at individual frequencies, air- and bone-conduction, Pure Tone Average (PTA) calculated on the side affected with VS based on hearing thresholds at $500 \mathrm{~Hz}, 1,000 \mathrm{~Hz}, 2,000 \mathrm{~Hz}$, and $3,000 \mathrm{~Hz}$ (this parameter is more commonly used in North America and recommended by the American Academy of Otolaryngology-Head and Neck Surgery (AAO-HNS)), ${ }^{1}$ interaural differences in the values of hearing thresholds for each of the test frequencies from $125 \mathrm{~Hz}$ to $8,000 \mathrm{~Hz}$, for air-conduction (the value of hearing threshold in the operated ear minus the value of hearing threshold in the non-operated ear), and interaural differences between PTA (the value of PTA in the operated ear minus the value of PTA in the non-operated ear). Moreover, the preoperative shape of the air-conduction audiometric curve was assessed, with the curve classified as normal, sharply falling, rising, flat, U-shaped, inverted U-shaped, and complete deafness.

The following parameters were assessed with speech audiometry in terms of being potentially prognostic factors: 1. speech discrimination for individual sound intensities with particular attention paid to the value of 55-65 dB of sound pressure level (dB SPL) as it corresponds with typical speech intensity; 2 . interaural differences in speech discrimination for individual values of sound intensity (speech discrimination for the non-operated minus speech discrimination for the operated ear at a specific intensity); 3. speech detection threshold (SDT) expressed in AB SPL; 4. speech reception threshold (SRT) - expressed in dB SPL; 5. speech discrimination score (SDS) - expressed as percentages; 6 . interaural difference between SDS (value for the non-operated ear minus value for the operated ear); 7. achieving $100 \%$ of speech discrimination by the patient - with the zero-one method.

Impedance audiometry was used to assess the presence or absence of stapedial reflex and the value of sound intensity at which stapedial reflex was obtained. However, if the reflex was absent, its value was recorded as $130 \mathrm{~dB}$ in order to facilitate the analyses. Moreover, the patients were grouped based on normal or pathological stapedial reflex (with the pathological reflex described as: test intensity 
exceeding reference values - $100 \mathrm{~dB}$ SPL, positive Metz symptom and complete lack of stapedial response).

Type A tympanograms were obtained in each patient. Therefore, tympanogram curve shape was disregarded as a prognostic factor.

Auditory brainstem response tests were conducted with the Smart Box platform integrated with Smart-EP software (Intelligent Hearing Systems, Corp., Miami, USA). A $90 \mathrm{~dB}$ nHL (decibels above normal adult hearing level) broadband click was used as an acoustic stimulus. The rate of stimulus delivery was $31 / \mathrm{s}$.

The following ABR testing parameters were assessed as possible prognostic factors: 1 . waveform morphology - the presence of waves I, III and V; 2. the value of wave I, III and V latency; 3. the values of I-III, I-V and III-V intervals; 4. interaural latency differences (ILD) for waves I, III and V; 5. ILD for I-III, I-V and III-V; 6. wave I, III and V amplitudes measured as the average of 3 records for each wave; 7. interaural amplitude differences for waves I, III and V; 8. interaural amplitude ratios of waves I, III and V - the value of amplitudes recorded on the side of the tumor and the value of contralateral amplitudes of individual waves; 9. amplitude ratios of waves V and I (ARI_V); 10. interaural ARI_V ratio - ipsilateral to the tumor vs contralateral side, compliance with referenced values according to Hall et al. ${ }^{2}$

Apart from the individual parameters for selected audiologic tests (pure-tone, speech, impedance audiometry, and $\mathrm{ABR}$ ) as possible prognostic factors of hearing preservation, we also assessed the preoperative hearing capacity calculated according to the AAO-HNS classification ${ }^{1}$ and the Classification of Hearing Impairments by the International Bureau for Audiophonology (BIAP). ${ }^{3}$

\section{Assessment of surgery-related hearing loss}

The abovementioned parameters of the assessment of pre- and postoperative hearing status, calculated on the basis of data obtained from pure-tone audiometry, were used to evaluate surgery-related hearing loss by determining absolute hearing loss. The majority of analyses in the present study refer to this parameter.

Absolute hearing loss was defined as a difference between PTA before and after the surgery for the operated ear.

\section{Statistical analysis}

All statistical calculations were performed with IBM SPSS statistical software (IBM Corp., Armonk, USA). Qualitative data was described with the use of the number and percentage of occurrence of a category, while quantitative variables were characterized with the following descriptive statistics: median (M), mean, standard deviation (SD), minimum (Min), and maximum (Max). Prior to analyses, all the quantitative variables had been examined in terms of data distribution with 2 measures of distribution: skewness and kurtosis. A result was considered statistically significant with a p-value below $0.05(\mathrm{p}<0.05)$. The following analyses were conducted in this study: $1 . \chi^{2}$ test in cross tabulations (combined with comparison of column proportions with Bonferroni correction); 2. correlation analysis (Pearson's $r$ / Spearman's rho); 3. polynomial logistic regression analysis; 4. k-means cluster analysis; 5 . one-way analysis of variance (ANOVA; intra-group and inter-group design).

\section{Ethical considerations}

The study was approved by the local Institutional Ethics Committee Review Board. The project conforms to the Code of Ethics of the World Medical Association (Declaration of Helsinki).

\section{Results}

\section{Study group division into 4 groups based on absolute hearing loss}

Based on absolute hearing loss (difference between PTA pre- and postoperatively for the operated ear), the patients were divided into 4 categories of differences: $\leq 10.00$; 10.01-20.00; 20.01-40.00; and $\geq 40.01$ (Table 1). The division aimed at distinguishing groups with low and high grades of hearing loss. This division into 4 groups became the basis for further analyses concerning possible factors influencing surgery-related hearing loss. The table presents the number and percentage of patients along with the average tumor size in each group.

Table 1. The division into groups based on surgery-related hearing loss, comprising the difference in Pure Tone Average (PTA; according to AAOHNS classification) before and after the surgery. The table presents number and percentage of patients along with average tumor size in each group

\begin{tabular}{|l|c|c|c|}
$\begin{array}{c}\text { Patient group } \\
\text { depending } \\
\text { on surgery-related } \\
\text { hearing loss [dB HL] }\end{array}$ & $\begin{array}{c}\text { Number } \\
\text { of patients }\end{array}$ & $\begin{array}{c}\text { Percentage } \\
\text { of patients } \\
{[\%]}\end{array}$ & $\begin{array}{c}\text { Average } \\
\text { tumor size } \\
\text { [mm] }\end{array}$ \\
\hline$\leq 10.00$ & 27 & 31.40 & 10.14 \\
\hline $10.01-20.00$ & 21 & 24.42 & 11.77 \\
\hline $20.01-40.00$ & 16 & 18.60 & 13.42 \\
\hline$\geq 40.01$ & 22 & 25.58 & 10.75 \\
\hline
\end{tabular}

\section{Pure-tone audiometry}

One-way ANOVA revealed a correlation between hearing thresholds for individual frequencies before the operation and preoperative PTA and the degree of surgery-related hearing loss. A thorough post hoc analysis with an S-N-K test showed that patients with hearing loss below $10 \mathrm{~dB} \mathrm{HL}$ (decibels hearing level) preserved considerably higher values of hearing threshold for the $1,000 \mathrm{~Hz}$ frequency than the remaining groups (a result at the level of statistical tendency 
Table 2. Pure Tone Averages (PTA) at individual frequencies and preoperative PTA [dB HL] depending on the extent of surgery-related hearing loss - statistically significant parameters

\begin{tabular}{|l|c|c|c|c|c|}
\multicolumn{1}{c}{$\begin{array}{c}\text { The parameter of } \\
\text { pure-tone audiometry }\end{array}$} & \multicolumn{4}{|c|}{ Hearing loss in individual groups [dB HL] } & \multicolumn{2}{|c|}{ F-test } \\
\cline { 2 - 5 } & $\leq 10.00$ & $10.01-20.00$ & $20.01-40.00$ & 140.01 \\
\hline Hearing threshold at $125 \mathrm{~Hz}$ & $24.07 \pm 15.81$ & $17.73 \pm 8.13$ & $14.67 \pm 6.67$ & $16.82 \pm 10.53$ \\
\hline Hearing threshold at $250 \mathrm{~Hz}$ & $25.56 \pm 16.49$ & $15.46 \pm 11.12$ & $15.33 \pm 9.90$ & $15.46 \pm 9.12$ \\
\hline Hearing threshold at $500 \mathrm{~Hz}$ & $28.33 \pm 20.33$ & $19.09 \pm 15.17$ & $17.67 \pm 15.34$ & $17.05 \pm 10.43$ \\
\hline Hearing threshold at $1,000 \mathrm{~Hz}$ & $38.33 \pm 24.22$ & $27.73 \pm 19.19$ & $25.33 \pm 21.25$ & $18.41 \pm 13.49$ \\
\hline
\end{tabular}

Table 3. Correlations between surgery-related hearing loss (the difference in pre- and postoperative Pure Tone Averages (PTA)) and the preoperative degree of hearing impairment (according to BIAP classification)

\begin{tabular}{|c|c|c|c|c|c|}
\hline \multirow{2}{*}{ Surgery-related hearing loss } & \multicolumn{4}{|c|}{ Preoperative degree of hearing impairment } & \multirow{2}{*}{ Total $[\%]$} \\
\hline & normal [\%] & slight [\%] & moderate [\%] & severe $[\%]$ & \\
\hline$\leq 10.00 \mathrm{~dB} \mathrm{HL}$ & $22.6^{a}$ & $24.0^{\mathrm{a}}$ & $42.9^{a, b}$ & $100.0^{b}$ & 31.40 \\
\hline $10.01-20.00 \mathrm{~dB} \mathrm{HL}$ & $25.8^{\mathrm{a}}$ & $20.0^{\mathrm{a}}$ & $32.1^{\mathrm{a}}$ & - & 25.60 \\
\hline $20.01-40.00 \mathrm{~dB} \mathrm{HL}$ & $16.1^{\mathrm{a}}$ & $20.0^{\mathrm{a}}$ & $17.9^{a}$ & - & 17.40 \\
\hline$\geq 40.01 \mathrm{~dB} \mathrm{HL}$ & $35.5^{\mathrm{a}}$ & $36.0^{\mathrm{a}}$ & $7.1^{b}$ & - & 25.60 \\
\hline Total & 100.00 & 100.00 & 100.00 & 100.00 & 100.00 \\
\hline
\end{tabular}

Each superscript letter (a,b) means a subset of "hearing impairment" category whose column proportions do not differ significantly at the level of 0.05.

was obtained only for $500 \mathrm{~Hz}$ frequency). The remaining differences revealed no statistical significance (Table 2).

The $\chi^{2}$ analysis in cross tabulations did not confirm any correlation between the type of audiogram and surgery-related hearing loss $\left(\chi^{2}=21.81 ; \mathrm{p}>0.05\right)$. It showed no correlation between surgery-related hearing loss (the difference between pre- and postoperative PTA) and the degree of BIAP hearing impairment $\left(\chi^{2}=13.62\right.$; $\left.\mathrm{p}>0.05\right)$. It is worth mentioning that the column proportions test with Bonferroni correction showed that the lowest degree of surgery-related hearing loss was obtained considerably more frequently in patients who had been preoperatively diagnosed with moderate or severe hearing impairment according to BIAP. ${ }^{3}$ Detailed results are presented in Table 3.

The $\chi^{2}$ analysis in cross tabulations showed no correlation between surgery-related hearing loss (the difference between pre- and postoperative PTA) and the preoperative hearing status described with the AAO-HNS classification $\left(\chi^{2}=4.62 ; \mathrm{p}>0.05\right)$.

\section{Speech audiometry}

Pearson's r correlation analysis was used to determine whether there was a correlation between the parameters of speech audiometry and the surgery-related hearing loss. The pre- and postoperative differences between PTA were compared in the analysis.

A correlation between the results of both tests was confirmed. It was demonstrated that the more marked the surgery-related hearing loss, the lower the preoperative SDT and SRT indexes found in the patients and the more patients achieved $100 \%$ of speech discrimination in the operated ear preoperatively.
The SDS parameter was found to have the weakest correlation with postoperative hearing loss. However, a trend was visible which showed that hearing loss experienced by patients as a result of surgery was more marked in the case of better preoperative speech discrimination. Detailed results are presented in Table 4.

Pearson's $\mathrm{r}$ calculated for the correlation between the percentage of speech discrimination at individual intensities and the surgery-related hearing loss showed a significant correlation for the intensities of $45 \mathrm{~dB}$ and higher, the surgery-related hearing loss increased with the increase of the percentage of speech discrimination for the intensities of $45 \mathrm{~dB}$ SPL and higher.

The results of Pearson's r correlation between preoperative interaural differences in speech discrimination during speech audiometry for individual intensities and surgeryrelated hearing loss showed a significant correlation for intensities above $50 \mathrm{~dB}$ SPL. Surgery-related hearing loss

Table 4. Correlations between the indexes of speech audiometry (speech reception threshold - SRT, speech detection threshold - SDT, peak percentage of speech discrimination in the operated ear - SDS, and whether the patient achieved $100 \%$ of speech discrimination - SDS100) and surgery-related hearing loss (the difference between pre- and postoperative Pure Tone Averages (PTA)) - the values of Pearson's r correlation coefficient

\begin{tabular}{|l|c|}
\multicolumn{1}{|c|}{ Index } & Surgery-related hearing loss \\
\hline SDT & $-0.324^{* *}$ \\
\hline SRT & $-0.341^{* *}$ \\
\hline SDS & 0.220 \\
\hline SDS100 & $0.325^{* *}$ \\
\hline
\end{tabular}

${ }^{*} p<0.05 ;{ }^{* *} p<0.01 ;{ }^{* *}$ statistically significant parameters. 
Table 5. Correlations between statistically significant preoperative indexes of speech audiometry for the operated ear and surgery-related hearing loss (the difference between pre- and postoperative Pure Tone Averages - PTA) - descriptive statistics ( \pm SD) and F statistics

\begin{tabular}{|c|c|c|c|c|c|c|}
\hline Speech audiometry parameters & $\begin{array}{l}\leq 10.00 \\
\mathrm{~dB} \mathrm{HL}\end{array}$ & $\begin{array}{c}10.01-20.00 \\
\mathrm{~dB} \mathrm{HL}\end{array}$ & $\begin{array}{c}20.01-40.00 \\
\text { dB HL }\end{array}$ & $\begin{array}{c}\geq 40.01 \\
\mathrm{~dB} \mathrm{HL}\end{array}$ & F-test & p-value \\
\hline SDT & $45.42 \pm 18.29$ & $40.00 \pm 17.24$ & $38.33 \pm 18.48$ & $30.26 \pm 10.07$ & 3.047 & 0.034 \\
\hline $55 \mathrm{~dB}$ SPL & $38.96 \pm 38.95$ & $47.00 \pm 40.80$ & $55.33 \pm 36.03$ & $70.00 \pm 25.66$ & 2.785 & 0.047 \\
\hline $60 \mathrm{~dB} S P L$ & $47.08 \pm 39.48$ & $54.75 \pm 39.69$ & $65.00 \pm 32.13$ & $78.68 \pm 22.48$ & 3.193 & 0.028 \\
\hline $65 \mathrm{~dB}$ SPL & $55.63 \pm 38.46$ & $61.25 \pm 36.41$ & $71.33 \pm 28.69$ & $83.95 \pm 19.19$ & 3.031 & 0.035 \\
\hline $70 \mathrm{~dB} S P L$ & $64.17 \pm 37.20$ & $68.25 \pm 33.57$ & $77.67 \pm 27.70$ & $89.21 \pm 18.20$ & 2.672 & 0.054 \\
\hline $75 \mathrm{~dB}$ SPL & $69.17 \pm 36.32$ & $73.50 \pm 26.81$ & $79.67 \pm 28.19$ & $91.32 \pm 15.97$ & 2.347 & 0.080 \\
\hline Interaural difference $25 \mathrm{~dB}$ SPL & $7.04 \pm 10.63$ & $-0.23 \pm 5.17$ & $1.67 \pm 9.94$ & $7.96 \pm 10.20$ & 4.056 & 0.010 \\
\hline Interaural difference $30 \mathrm{~dB}$ SPL & $12.69 \pm 22.11$ & $-0.68 \pm 10.27$ & $2.67 \pm 20.34$ & $13.41 \pm 17.42$ & 3.344 & 0.023 \\
\hline Interaural difference $35 \mathrm{~dB}$ SPL & $22.22 \pm 28.31$ & $4.43 \pm 20.40$ & $19.33 \pm 30.79$ & $20.34 \pm 19.08$ & 2.453 & 0.069 \\
\hline
\end{tabular}

Table 6. The distribution of mean values and standard deviation (SD) of statistically significant parameters in 4 individual groups of surgery-related hearing loss

\begin{tabular}{|c|c|c|c|c|c|c|}
\hline Parameter & $\begin{array}{l}\leq 10.00 \\
\mathrm{~dB} \mathrm{HL}\end{array}$ & $\begin{array}{c}10.01-20.00 \\
\text { dB HL }\end{array}$ & $\begin{array}{c}20.01-40.00 \\
\text { dB HL }\end{array}$ & $\begin{array}{c}\geq 40.01 \\
\mathrm{~dB} \mathrm{HL}\end{array}$ & F-test & p-value \\
\hline \multicolumn{7}{|c|}{ Pure-tone audiometry } \\
\hline Hearing threshold at $125 \mathrm{~Hz}$ & $24.07 \pm 15.81$ & $17.73 \pm 8.13$ & $14.67 \pm 6.67$ & $16.82 \pm 10.53$ & 2.817 & 0.044 \\
\hline Hearing threshold at $250 \mathrm{~Hz}$ & $25.56 \pm 16.49$ & $15.46 \pm 11.12$ & $15.33 \pm 9.90$ & $15.46 \pm 9.12$ & 4.067 & 0.010 \\
\hline Hearing threshold at $500 \mathrm{~Hz}$ & $28.33 \pm 20.33$ & $19.09 \pm 15.17$ & $17.67 \pm 15.34$ & $17.05 \pm 10.43$ & 2.634 & 0.055 \\
\hline Hearing threshold at $1,000 \mathrm{~Hz}$ & $38.33 \pm 24.22$ & $27.73 \pm 19.19$ & $25.33 \pm 21.25$ & $18.41 \pm 13.49$ & 4.133 & 0.009 \\
\hline \multicolumn{7}{|c|}{ Speech audiometry } \\
\hline SDT & $45.42 \pm 18.29$ & $40.00 \pm 17.24$ & $38.33 \pm 18.48$ & $30.26 \pm 10.07$ & 3.047 & 0.034 \\
\hline $65 \mathrm{~dB}$ SPL & $55.63 \pm 38.46$ & $61.25 \pm 36.41$ & $71.33 \pm 28.69$ & $83.95 \pm 19.19$ & 3.031 & 0.035 \\
\hline $70 \mathrm{~dB} S P L$ & $64.17 \pm 37.20$ & $68.25 \pm 33.57$ & $77.67 \pm 27.7$ & $89.21 \pm 18.20$ & 2.672 & 0.054 \\
\hline $75 \mathrm{~dB}$ SPL & $69.17 \pm 36.32$ & $73.50 \pm 26.81$ & $79.67 \pm 28.19$ & $91.32 \pm 15.97$ & 2.347 & 0.080 \\
\hline Interaural difference $25 \mathrm{~dB}$ SPL & $7.04 \pm 10.63$ & $-0.23 \pm 5.17$ & $1.67 \pm 9.94$ & $7.96 \pm 10.20$ & 4.056 & 0.010 \\
\hline Interaural difference $30 \mathrm{~dB}$ SPL & $12.69 \pm 22.11$ & $-0.68 \pm 10.27$ & $2.67 \pm 20.34$ & $13.41 \pm 17.42$ & 3.344 & 0.023 \\
\hline Interaural difference $35 \mathrm{~dB}$ SPL & $22.22 \pm 28.31$ & $4.43 \pm 20.40$ & $19.33 \pm 30.79$ & $20.34 \pm 19.08$ & 2.453 & 0.069 \\
\hline \multicolumn{7}{|c|}{$A B R$} \\
\hline III-V interval & $2.09 \pm 0.44$ & $1.88 \pm 0.15$ & $1.74 \pm 0.24$ & $2.22 \pm 0.49$ & 4.701 & 0.005 \\
\hline Wave V amplitude & $0.23 \pm 0.09$ & $0.30 \pm 0.10$ & $0.23 \pm 0.10$ & $0.22 \pm 0.10$ & 3.129 & 0.030 \\
\hline Interaural difference of III-V intervals & $0.34 \pm 0.41$ & $0.12 \pm 0.08$ & $0.33 \pm 0.30$ & $0.41 \pm 0.37$ & 2.618 & 0.059 \\
\hline \multicolumn{7}{|c|}{ Impedance audiometry } \\
\hline Reflex normality for $500 \mathrm{~Hz}$ in the operated ear & $0.30 \pm 0.47$ & $0.71 \pm 0.46$ & $0.67 \pm 0.49$ & $0.76 \pm 0.44$ & 4.313 & 0.008 \\
\hline Reflex normality for $1,000 \mathrm{~Hz}$ in the operated ear & $0.22 \pm 0.42$ & $0.52 \pm 0.51$ & $0.5 \pm 0.52$ & $0.71 \pm 0.47$ & 3.643 & 0.017 \\
\hline Reflex normality for $2,000 \mathrm{~Hz}$ in the operated ear & $0.09 \pm 0.29$ & $0.33 \pm 0.48$ & $0.33 \pm 0.49$ & $0.53 \pm 0.51$ & 3.392 & 0.023 \\
\hline
\end{tabular}

increased with a more marked interaural diversity for intensities above $50 \mathrm{~dB}$ SPL. The same tendency occurred for the interaural difference of SDS parameters. Therefore, patients who had better preoperative speech discrimination in the operated ear compared to the non-operated ear experienced more marked surgery-related hearing loss.

One-way ANOVA was used to determine if there was a correlation between the indexes of the speech audiometry test and surgery-related hearing loss with the division into 4 groups (Table 5). Significant differences were only obtained for the SDT $(\mathrm{p}<0.05)$ in the operated ear.
A detailed SDT analysis showed that the only diversity was observed between groups of patients with the lowest and the most marked surgery-related hearing loss. The highest results were obtained in the group with the lowest hearing loss and the mean SDT value in this group was $45 \mathrm{~dB}$ SPL. Patients who lost over $40 \mathrm{~dB}$ HL of PTA had had the mean preoperative SDT value of $30 \mathrm{~dB}$ SPL. We also observed a trend showing that SDT values decreased with increased surgery-related hearing loss (Table 6).

The analysis of speech discrimination percentage for individual speech intensities showed significant differences 
in regard to surgery-related hearing loss observed for the $55 \mathrm{~dB}$ to $75 \mathrm{~dB}$ SPL band, which includes the key intensity for interpersonal communication and $60 \mathrm{~dB}$ SPL band, which is typical for conversation (depending on the source: 55-65 dB SPL).

The analysis of interaural difference of speech discrimination for individual intensities confirmed the presence of differences in interaural difference of speech discrimination only for the intensities from $25 \mathrm{~dB}$ to $35 \mathrm{~dB}$ SPL. A thorough post hoc analysis with $\mathrm{S}-\mathrm{N}-\mathrm{K}$ test showed that patients with surgery-related hearing loss from $10.01 \mathrm{~dB}$ to $40.00 \mathrm{~dB} \mathrm{HL}$ achieved considerably lower results of the analyzed indexes than the remaining groups (Table 6). The only exception in the case of interaural difference in speech discrimination for $35 \mathrm{~dB}$ SPL was noted in patients with the difference in pure-tone audiometry of 10.01-20.00 dB HL. The remaining differences revealed no statistical significance. The analysis proved that the patients more commonly experienced moderate surgery-related hearing loss (10-40 dB HL of PTA) if their speech discrimination on the side of the tumor was better and similar to speech discrimination on the non-operated side for the intensities of 25-35 dB SPL.

No intergroup differences were observed in case of the remaining parameters.

\section{Auditory brainstem response testing}

Pearson's r correlation analysis demonstrated an increasing surgery-related hearing loss in the operated ear along with the growing deviation of I-V and III-V intervals from the norm, regardless of the PTA index investigated. It was also demonstrated that the presence of wave $\mathrm{V}$ in the operated ear in ABR waveform morphology correlated with lower surgery-related hearing loss. Moreover, the analyses revealed that the hearing loss resulting from the surgery was more marked if the I-V interval presented higher values in the operated ear. Additionally, a negative correlation was observed between surgery-related hearing loss and the presence of wave $\mathrm{V}$. Therefore, patients whose waveform morphology lacked wave $\mathrm{V}$ experienced a more marked surgery-related hearing loss. The remaining correlations revealed no statistical significance.

One-way ANOVA between ABR parameters and surgeryrelated hearing loss in the 4 groups demonstrated significant differences for the value of III-V interval $(\mathrm{p}<0.01)$. A detailed post hoc analysis with S-N-K test showed that patients with the most abundant hearing loss ( $\geq 40.01 \mathrm{~dB} H \mathrm{HL})$ achieved considerably higher values of III-V interval before the surgery than patients with a surgery-related PTA reduction ranging from $10.01 \mathrm{~dB}$ to $40.00 \mathrm{~dB} \mathrm{HL}$. The lowest values of III-V interval were achieved if PTA reduction ranged from $20.01 \mathrm{~dB}$ to $40.00 \mathrm{~dB}$ HL. No difference was observed between patients with the lowest and the most abundant surgery-related hearing loss. The resultant relationship is presented in Table 6. A significant difference was also found as regards the value of wave $\mathrm{V}$ amplitude $(\mathrm{p}<0.05) . \mathrm{S}-\mathrm{N}-\mathrm{K}$ test analysis revealed that the subjects with a pre- vs postoperative difference between PTA ranging from $10.01 \mathrm{~dB}$ to $20.00 \mathrm{~dB}$ HL had markedly higher values of preoperative wave $\mathrm{V}$ amplitude compared to the remaining groups $(p=0.050)$. The remaining differences revealed no statistical significance. Differences at the level of statistical tendency ( $p=0.059$ ) were obtained for the interaural difference of III-V intervals. A detailed post hoc analysis with $\mathrm{S}-\mathrm{N}-\mathrm{K}$ test revealed that the patients with a pre- vs postoperative difference between PTA ranging from $10.01 \mathrm{~dB}$ to $20.00 \mathrm{~dB}$ HL had markedly lower values of interaural difference of III-V intervals compared to the remaining subjects.

The $\chi^{2}$ test in cross tabulations also indicated a significant relationship in the assessment of normal values of III-V interval in the operated ear $\left(\chi^{2}=9.06 ; \mathrm{p}<0.05\right)$. A thorough analysis of column comparisons with Bonferroni correction showed that III-V interval was more commonly normal in the group with surgery-related hearing loss ranging from $10.01 \mathrm{~dB}$ to $40.00 \mathrm{~dB}$ HL compared to the subjects with surgery-related hearing loss below $10 \mathrm{~dB}$ HL or over $40 \mathrm{~dB}$ HL. The remaining ABR parameters revealed no statistical significance.

\section{Impedance audiometry}

One-way ANOVA demonstrated significant differences for a parameter predefined as normal stapedial reflex at $500 \mathrm{~Hz}$ on the operated side ( $<$ 0.01). A detailed S-N-K analysis showed that the subjects with surgery-related hearing loss lower than $10 \mathrm{~dB}$ HL significantly more often had an abnormal ipsilateral stapedial reflex at the frequency of $500 \mathrm{~Hz}$ in the operated ear compared to the remaining groups of patients (Table 6).

Moreover, significant differences were observed for the normality of ipsilateral stapedial reflex at $1,000 \mathrm{~Hz}$ and $2,000 \mathrm{~Hz}$ on the operated side $(\mathrm{p}<0.05)$. S-N-K test analysis revealed significant differences between groups with the lowest and the most severe surgery-related hearing loss. Normal stapedial reflexes were observed in the group with surgery-related hearing loss of over $40 \mathrm{~dB}$ HL. The differences between the remaining groups revealed no statistical significance.

Significant differences at the statistical trend level were obtained for the index of stapedial reflex at the frequency of 4,000 Hz for the contralateral non-operated ear $(\mathrm{p}=0.085)$. The obtained results indicate the presence of a negative correlation - the value of the parameter decreased with the increased surgery-related hearing loss.

The $\chi^{2}$ analysis in cross tabulations was used to determine if there was any correlation between the normality of stapedial reflex (at the frequencies of $500 \mathrm{~Hz}, 1,000 \mathrm{~Hz}$, $2,000 \mathrm{~Hz}$, and $4,000 \mathrm{~Hz}$ ) in the operated ear (ipsi) and surgery-related hearing loss. A correlation was confirmed for reflex normality at $500 \mathrm{~Hz}\left(\chi^{2}=11.53\right.$; $\left.\mathrm{p}<0.01\right)$. A thorough analysis of column comparisons with Bonferroni 
correction showed a significantly lower percentage of reflex normality at $500 \mathrm{~Hz}$ in the operated ear in the group with the lowest surgery-related hearing loss in comparison to the remaining groups. The analysis also confirmed a correlation for reflex normality at the frequency of $1,000 \mathrm{~Hz}$ $\left(\chi^{2}=9.98 ; \mathrm{p}<0.05\right)$ and $2,000 \mathrm{~Hz}\left(\chi^{2}=9.38 ; \mathrm{p}<0.05\right)$. A thorough analysis of column comparisons with Bonferroni correction showed a significantly lower percentage of reflex normality at $1,000 \mathrm{~Hz}$ in the group with the lowest surgery-related hearing loss in comparison with the remaining groups (except the group in which puretone audiometry revealed differences between 20.01 dB and $40.00 \mathrm{~dB})$. However, no correlation was found for reflex normality at $4,000 \mathrm{~Hz}\left(\chi^{2}=3.11 ; \mathrm{p}<0.05\right)$. It is worth emphasizing that the results obtained match the trend observed for reflex normality at $500 \mathrm{~Hz}, 1,000 \mathrm{~Hz}$ and $2,000 \mathrm{~Hz}$. Pearson's r correlation analysis confirmed a correlation between the indexes of impedance audiometry and surgery-related hearing loss (pre- and postoperative differences between PTA). The analysis revealed an increase in absolute surgery-related hearing loss with the increasing reflex normality at $500 \mathrm{~Hz}, 1,000 \mathrm{~Hz}$ and 2,000 Hz. Moreover, surgery-related hearing loss became more marked with the reduction in the values of preoperative parameters of impedance audiometry, such as ipsilateral sound intensity for the stapedial reflex in the operated ear at the frequency of $2,000 \mathrm{~Hz}$, and contralateral sound intensity for stapedial reflex in the non-operated ear at the frequencies of $500 \mathrm{~Hz}, 1,000 \mathrm{~Hz}, 2,000 \mathrm{~Hz}$, and 4,000 Hz. The remaining correlations revealed no statistical significance.

\section{Discussion}

The present study analyzes the problem of hearing preservation after VS removal at a slightly different angle than available professional literature concerning this topic. In principle, previous studies classified patients according to various scales to assess the initial hearing status on a 3to 5-grade scale according to which it was stated whether the hearing status improved, deteriorated or remained at the same level. ${ }^{1,4-14}$

The present study concentrates on the assessment of absolute hearing loss in order to identify the factors which influence the absolute value of "lost decibels" in pure-tone audiometry, and, what follows, the factors which determine the preservation or loss of initial hearing status irrespective of the preoperative status. The problem of "hearing preservation" was specifically approached in this study - to preserve hearing as similar as possible to the preoperative status. The study did not exclusively concern the issue of serviceable hearing criteria which may differ to a large extent depending on an implemented scale. Dugar et al. and Lassaletta et al. observed that the preservation of serviceable hearing may range from $0 \%$ to $56 \%$ depending on the selected scale. ${ }^{15,16}$
It is worth emphasizing that no scales were used to calculate the results in this study. Surgery-related hearing loss was reviewed objectively, exclusively on the basis of study group analysis. At the same time, our intention was not to use the described techniques of determining serviceable hearing and not to use scales (largely based on speech audiometry) for the main calculations. Nonetheless, a certain amount of data concerning not only hearing sounds but also sound perception as speech are not available. However, the aim of this study was not to repeat available studies, but to focus on a new approach to the topic.

To the best of our knowledge, detailed analyses concerning the possible prognostic audiologic factors of hearing preservation in patients with VS have not been published so far.

\section{Pure-tone audiometry}

The present study demonstrated that surgery-related hearing loss is influenced by BIAP hearing impairment degree determined before the surgery. ${ }^{3}$ The lowest degree of surgery-related hearing loss, below $10 \mathrm{~dB}$ HL of PTA, was obtained considerably more frequently in patients who had been preoperatively diagnosed with moderate or severe hearing impairment. Additionally, it was demonstrated that the most marked surgery-related hearing loss was observed in patients with previously normal hearing status.

Ferber-Viart et al. assessed the prognostic value of selected parameters of pure-tone audiometry for hearing preservation in patients operated on due to VS. ${ }^{17}$ Hearing preservation was defined by the maintenance of AAO-HNS hearing status grade $\mathrm{A}, \mathrm{B}$ or $\mathrm{C}$. The assessment covered the hearing thresholds for individual frequencies: $250 \mathrm{~Hz}$, $500 \mathrm{~Hz}, 1,000 \mathrm{~Hz}, 2,000 \mathrm{~Hz}, 4,000 \mathrm{~Hz}$, and 8,000 Hz, and PTA for the following frequencies: $500 \mathrm{~Hz}, 1,000 \mathrm{~Hz}$, $2,000 \mathrm{~Hz}$, and 4,000 Hz. Statistical significance was confirmed for hearing threshold values at $250 \mathrm{~Hz}, 4,000 \mathrm{~Hz}$ and $8,000 \mathrm{~Hz}$, and PTA. The authors stated that the possibility of hearing preservation significantly decreased above the value of $50 \mathrm{~dB}$ HL PTA. According to Rohit et al., preoperative PTA markedly contributes to surgery-related hearing loss. ${ }^{18}$ However, the value of postoperative PTA negatively correlated with the size of the tumor. Similar correlations were not confirmed by a study by Slattery et al. ${ }^{19}$ and Brackmann et al., ${ }^{20}$ as well as the results of the present analyses. Rastogi et al. analyzed the influence of preoperative hearing thresholds at $4,000 \mathrm{~Hz}$ and $8,000 \mathrm{~Hz}$ on surgery-related hearing status in patients operated on due to VS. ${ }^{21}$ However, no statistically significant correlations were demonstrated.

\section{Speech audiometry}

The present study revealed a trend showing that preoperative SDT values decreased with increased surgery-related hearing loss. Based on statistical analyses, patients with SDT $\geq 45 \mathrm{~dB}$ SPL are expected to lose $\leq 10 \mathrm{~dB}$ HL of PTA as a result of the surgery, while patients with SDT $\leq 30 \mathrm{~dB}$ 
SPL are at risk of the most marked surgery-related hearing loss (loss of $>40 \mathrm{~dB}$ HL of PTA). The analysis of the influence of speech discrimination for individual intensities on surgery-related hearing loss, both with and without the division into 4 groups, showed that surgery-related hearing loss increased with the percentage of speech discrimination for individual intensities. Moreover, correlation analysis demonstrated that surgery-related hearing loss significantly increased with the growing percentage of speech discrimination for the intensities of $45 \mathrm{~dB}$ SPL and above. Significant differences concerning surgeryrelated hearing loss in the specified 4 groups were observed for speech discrimination for the $55 \mathrm{~dB}$ to $75 \mathrm{~dB}$ SPL band, which includes the key intensity band for interpersonal communication typical for conversation (55-65 dB SPL). The present study results may indicate that the surgery affects those parameters of hearing which appear to be critical for interpersonal communication and a similar physical nerve fiber destruction is responsible for a disproportionately large reduction in perception. The issue requires confirmation in further research because of the lack of other publications in this field.

The analysis of interaural differences in speech discrimination for individual intensities and surgery-related hearing loss without the division into 4 groups showed that surgery-related hearing loss became more marked with increasing interaural diversity for intensities above $50 \mathrm{~dB}$ SPL. The division into 4 groups additionally showed that the better the speech audiometry result in the operated ear for the intensities of 25-35 dB SPL, the more frequently the patients experienced moderate surgery-related hearing loss (10-40 dB HL PTA).

Therefore, the analyses of speech audiometry results showed that patients who had had better preoperative speech discrimination in the operated ear vs non-operated ear experienced more marked surgery-related hearing loss. The review of the professional literature revealed that speech audiometry parameters including speech detection threshold (SDT), speech reception threshold (SRT) and speech discrimination score (SDS) were most commonly assessed in terms of their influence on hearing loss. According to some previous research, mean preoperative SDS was estimated at $66-96 \% .8,9,18,22,23$

Shelton et al. ${ }^{8}$ stated that SDT and SDS did not affect hearing status postoperatively, while 10 years later a similar study conducted by Ferber-Viart et al. ${ }^{17}$ revealed a significant influence of SDT on hearing preservation postoperatively. Moreover, it was stated that $45 \mathrm{~dB}$ SPL was the SDT level above which patients experienced a marked surgery-related deterioration of hearing. The present study also indicated no influence of SDS on postoperative hearing status. Rohit et al. also found no correlation between SDS and surgery-related hearing loss. ${ }^{18}$ Rastogi et al. conducted a study including 44 VS patients which demonstrated no influence of SRT or SDS on postoperative hearing status ${ }^{21}$.

\section{Auditory brainstem response testing}

The present analyses which did not comprise the division into groups demonstrated increasing surgery-related hearing loss in the operated ear along with a growing deviation of I-V and III-V intervals from the reference values, regardless of the PTA parameter investigated. Moreover, the analyses revealed that the hearing loss resulting from the surgery was more marked if the I-V interval presented higher values in the operated ear (according to $\mathrm{BIAP}^{3}$ ).

Additionally, a negative correlation was observed between surgery-related hearing loss and the presence of wave V. Therefore, patients whose waveform morphology lacked wave $\mathrm{V}$ experienced a more marked surgeryrelated hearing loss. Additionally, the analysis of surgeryrelated hearing loss including the division into 4 groups showed that patients in whom hearing loss was the most marked ( $\geq 40.01 \mathrm{~dB} \mathrm{HL}$ ) achieved considerably higher values of III-V interval before the surgery. It was also demonstrated that the subjects with a pre- vs postoperative difference between PTA ranging from $10.01 \mathrm{~dB}$ to $20.00 \mathrm{~dB} \mathrm{HL}$ achieved markedly higher values of preoperative wave $\mathrm{V}$ amplitude compared to the remaining groups.

The analysis of the normality of selected ABR parameters in terms of reference values according to Hall et al. in 4 selected groups demonstrated that III-V interval was more commonly normal compared to subjects whose surgery-related hearing loss was below $10 \mathrm{~dB}$ HL or above $40 \mathrm{~dB}$ HL. ${ }^{2}$ This may indicate that patients with the abnormal value of III-V interval had poorer conduction of bioelectric arousal, and what follows, poorer hearing. Therefore, surgery-related hearing loss was not as marked. However, this requires further study due to the fact that hearing loss is the most marked in such patients. It was demonstrated that surgery-related hearing loss was not affected by the normality of parameters like I and $V$ latency for the operated and non-operated ear, I-III intervals for the operated and non-operated ear, I-V intervals for the operated and non-operated ear, interaural latency difference for wave $\mathrm{V}$, and the amplitude ratio of waves I and $V$ for the operated and non-operated ear.

Additionally, cluster analysis demonstrated that surgery-related hearing loss most commonly ranged between $10.01 \mathrm{~dB}$ and $40.00 \mathrm{~dB}$ HL in patients with normal ABR parameters according to Hall's reference values, ${ }^{2}$ while patients whose ABR parameters were abnormal most commonly lost over $40 \mathrm{~dB}$ HL. This was consistent with the tendency identified in the present study which was observed in all audiologic tests and indicated that the absolute surgery-related hearing loss was more marked if the preoperative hearing status was better.

Ferber-Viart et al. analyzed ABR recordings in terms of their prognostic value for the postoperative hearing status in order to assess the presence of waves I, III and V, their latency and the duration of I-V interval. ${ }^{17}$ The study demonstrated that wave III was present in waveform morphology 
and wave $\mathrm{V}$ latency was over $6.5 \mathrm{~ms}$ in a group with preserved postoperative hearing, which was defined by the authors as the preservation of hearing at the level of AAOHNS class A, B or C. ${ }^{1}$ Matthies and Samii analyzed 420 preoperative ABRs and proved that the presence of waves I, III and V in waveform morphology correlated with a higher SDS in postoperative speech audiometry. ${ }^{24}$ Shelton et al. confirmed a negative correlation of postoperative SDS with abnormal interaural latency difference for wave V. ${ }^{8}$ Patients with a value of interaural latency difference for wave $\mathrm{V}$ not exceeding $0.4 \mathrm{~ms}$ preserved hearing at a rate of $78 \%$ (according to the authors' own classification). In the case of values ranging from $0.5 \mathrm{~ms}$ to $2 \mathrm{~ms}$, the percentage of patients with preserved hearing decreased to $58 \%$.

Browning et al. conducted a study on a group of $36 \mathrm{pa}-$ tients operated on with a hearing preservation technique. ${ }^{25}$ They demonstrated a correlation between preserved intraoperative ABR waveform morphology and a slight loss in postoperative PTA. However, the correlation revealed no statistical significance. Auditory brainstem response waveform morphology as a factor contributing to hearing loss was undermined by a study conducted by Kanzaki et al., who analyzed the recordings of 27 patients who had undergone hearing preservation surgery. ${ }^{26}$ They demonstrated a correlation between the presence of wave $V$ and the interaural latency difference for wave $\mathrm{V}$ vs postoperative hearing preservation, but the factors were not statistically significant.

A study including 71 patients was conducted by Dornhoffer et al., who demonstrated a statistically significant correlation between wave V latency and low surgery-related hearing loss. ${ }^{23}$ The patients with results not exceeding $6.8 \mathrm{~ms}$ had significantly better hearing after the surgery. However, interaural latency difference for wave $\mathrm{V}$ appeared to have no influence on postoperative hearing status.

A study including 104 patients conducted by Rohit et al. showed no statistically significant difference in regard to ABR waveform morphology, I-V interval and interaural latency difference for wave $\mathrm{V}$ between a group with preserved hearing and a group with surgery-related hearing loss. ${ }^{18}$ Vincent et al. conducted a study in which they assessed hearing after VS removal in 77 patients. ${ }^{27}$ They demonstrated that prolonged wave $\mathrm{V}$ latency in preoperative ABR recording is linked to a poorer prognosis regarding hearing status. Gardener et al. emphasized that even the preoperative lack of ABR data was not a factor which negatively correlated with postoperative hearing preservation. ${ }^{10}$ However, the study was conducted on a small sample size (9 subjects). Therefore, it requires a confirmation by research covering a larger population.

\section{Impedance audiometry}

The analysis of the normality of the stapedial reflex conducted in the present study, with the study group divided into patients with a normal and pathological reflex, demonstrated a trend which indicates that normal stapedial reflex was correlated with more marked hearing loss and a pathological reflex - with a less marked hearing loss. This was probably due to the initial hearing status on the side of the tumor and remained consistent with a tendency demonstrated in the present paper indicating that more marked absolute surgery-related hearing loss was observed in patients with better preoperative hearing status.

Berrettini et al. analyzed a pathological reflex as abnormal reflex, the lack of stapedial reflex and a positive Metz test result in patients with VS only at 1 frequency of $1,000 \mathrm{~Hz} .{ }^{28}$ Abnormal reflex was found in $59.5 \%$ of patients, which is consistent with the present study group (53\%) and confirms a proper selection of patients for further analyses.

This study shows that the subjects with surgery-related hearing loss lower than $10 \mathrm{~dB}$ HL significantly more often had an abnormal ipsilateral stapedial reflex at the frequency of $500 \mathrm{~Hz}$ in the operated ear compared to the remaining groups of patients. Regarding the frequency of $1,000 \mathrm{~Hz}$, it was demonstrated that the patients with a normal stapedial reflex significantly more commonly experienced major hearing loss (over $40 \mathrm{~dB}$ HL of PTA). Analogous results were also obtained for the frequency of $2,000 \mathrm{~Hz}$.

A significant correlation was observed between surgeryrelated hearing loss and intensity value for the contralateral stapedial reflex at the frequency of $2,000 \mathrm{~Hz}$ for the non-operated ear. Considerably lower intensities were obtained in the group where the absolute hearing loss was the most marked (PTA over $40 \mathrm{~dB} \mathrm{HL}$ ), and the highest intensities were obtained in the group where surgery-related hearing loss was the least marked (PTA below $10 \mathrm{~dB} \mathrm{HL}$ ).

Moreover, surgery-related hearing loss increased with a reduction in the values of some preoperative parameters of impedance audiometry. This also indicates that a higher risk of marked absolute hearing loss did not only correlate with better hearing status.

To the best of our knowledge, no professional literature is available which would cover the topic of possible prognostic factors of hearing preservation regarding impedance audiometry.

\section{Conclusions}

Univariate analyses of audiologic results, such as puretone audiometry, speech audiometry, ABR test, and impedance audiometry coherently demonstrated that the better the preoperative hearing, the more substantial surgeryrelated hearing loss was observed.

The following preoperative audiologic parameters were demonstrated to have a prognostic value for the extent of surgery-related hearing loss in patients with vestibular schwannoma (Table 7): 1. pure-tone audiometry parameters such as: hearing threshold at $125 \mathrm{~Hz}, 500 \mathrm{~Hz}, 1,000 \mathrm{~Hz}$, 
Table 7. Statistically significant $(p<0.5)$ parameters influencing the extent of hearing loss following VS removal

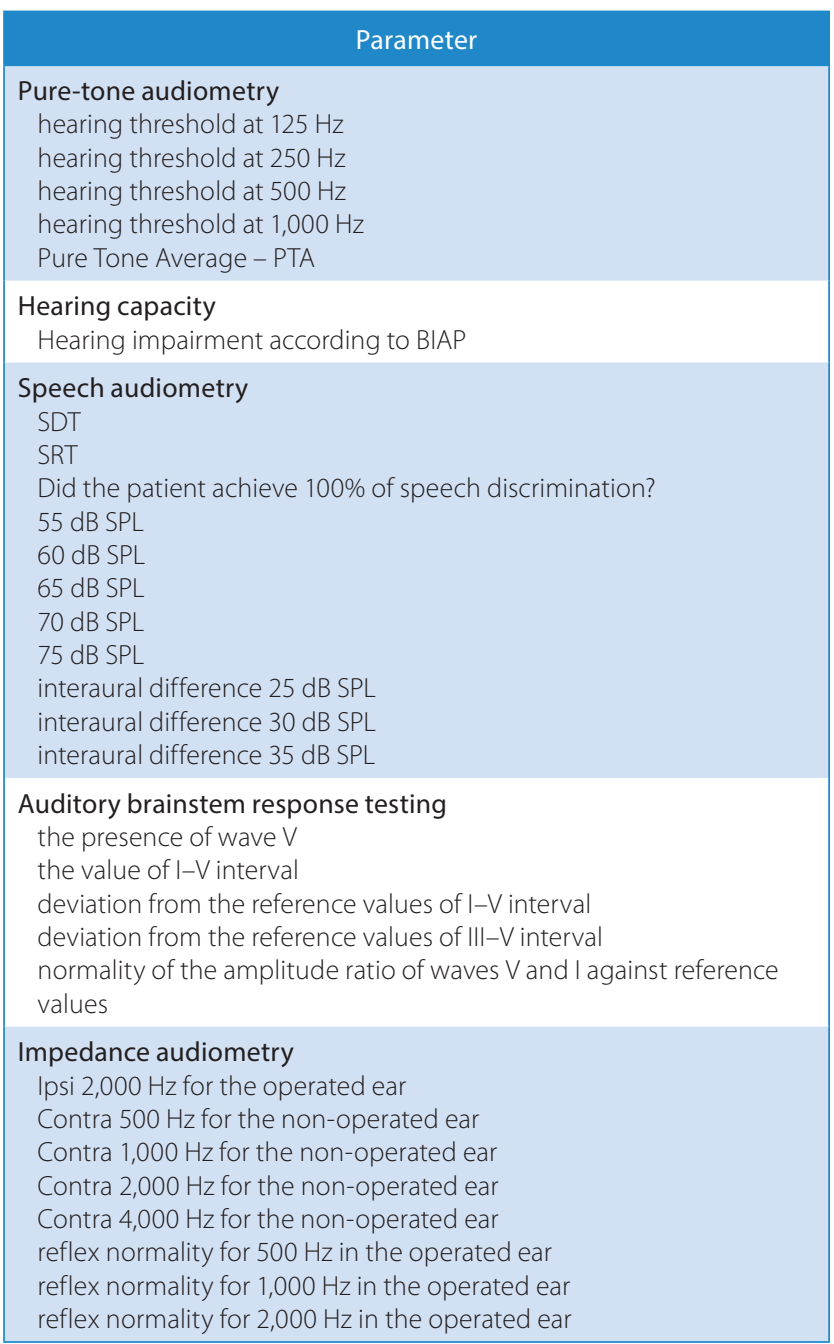

and PTA for the affected ear; 2. speech audiometry parameters such as: speech discrimination between $55 \mathrm{~Hz}$ and $75 \mathrm{~dB}$ SPL, SDT for the affected ear, interaural difference in speech discrimination between $25 \mathrm{~dB}$ and $35 \mathrm{~dB}$ SPL; 3. speech audiometry showed that the surgery mostly affects those parameters of hearing which appear to be critical for interpersonal communication (speech intensity of $60 \mathrm{~dB}$ SPL) and a similar physical nerve fiber destruction is responsible for a disproportionately large reduction in perception compared to the perception of a different range of speech intensity; 4 . auditory brainstem response test parameters such as: the presence of $\mathrm{V}$ wave, the values of I-V and III-V intervals and the amplitude of wave V; 5. impedance audiometry with parameters such as intensity level for obtaining stapedial reflex or an abnormal reflex at Ipsi $500 \mathrm{~Hz}, 1,000 \mathrm{~Hz}$ and 2,000 Hz, and Contra $500 \mathrm{~Hz}, 1,000 \mathrm{~Hz}, 2,000 \mathrm{~Hz}$, and 4,000 Hz.

\section{References}

1. AAO-HNS. Committee on Hearing and Equilibrium guidelines for the evaluation of hearing preservation in acoustic neuroma (vestibular schwannoma). American Academy of Otolaryngology-Head and Neck Surgery Foundation, INC. Otolaryngol Head Neck Surg. 1995; 113(3):179-180. doi:10.1016/S0194-5998(95)70101-X

2. Hall JW III, Mueller HG III, eds. Audiologists'Desk Reference: Volume I. Diagnostic Audiology Procedures and Practices. San Diego, CA: Singular Publishing Group; 1997.

3. International Bureau for Audiophonology BIAP. Recommendation 02/1 bis - Audiometric classification of hearing impairments. www. biap.org. https://www.biap.org/en/recommandations/recommendations/tc-02-classification. Published October 26, 1996. Updated July 10, 2017. Accessed July 21, 2017.

4. Sanna M, Khrais T, Piccirillo E, Russo A, Augurio A. Hearing preservation surgery in vestibular schwannoma: The hidden truth. Ann Otol Rhinol Laryngol. 2004;113(2):156-163.

5. Samii M, Matthies C. Management of 1000 vestibular schwannomas (acoustic neuromas). The facial nerve: Preservation and restitution of function. Neurosurgery. 1997;40(4):684-695.

6. Kanzaki J, Tos M, Sanna M, Moffat DA. New and modified reporting systems from the consensus meeting on systems for reporting results in vestibular schwannoma. Otol Neurotol. 2003;24(4):642-649.

7. Kanzaki J, Kunihiro T, Inoue Y. Some proposals on reporting systems in acoustic neuroma. In: Kanzaki J, ed. Acoustic Neuroma: Consensus on Systems for Reporting Results. Keio University International Symposia for Life Sciences and Medicine. Vol 10. Tokyo-New York: Springer; 2003:107-112.

8. Shelton C, Brackmann DE, House WF, Hitselberger WE. Acoustic tumor surgery: Prognostic factors in hearing conservation. Arch Otol Head Neck Surg. 1989;115(10):1213-1216.

9. Hecht CS, Honrubia VF, Wiet RJ, Sims HS. Hearing preservation after acoustic neuroma resection with tumor size used as a clinical prognosticator. Laryngoscope. 1997;107(8):1122-1126.

10. Gardner G, Robertson JH. Hearing preservation in unilateral acoustic neuroma surgery. Ann Otol Rhinol Laryngol. 1988;97(1):55-66.

11. Koos WT, Day JD, Matula C, Levy DI. Neurotopographic considerations in the microsurgical treatment of small acoustic neurinomas. J Neurosurg. 1998;88(3):506-512.

12. Caye-Thomasen P, Dethloff T, Hansen S, Stangerup S-E, Thomsen J. Hearing in patients with intracanalicular vestibular schwannomas. Audiol Neurotol. 2007;12(1):1-12.

13. Kanzaki J, ed. Acoustic Neuroma: Consensus on Systems for Reporting Results. Keio University International Symposia for Life Sciences and Medicine. Springer. 2003;10:183-192.

14. Fukushima T. Patient selection and method of evaluation for hearing preservation in acoustic neuroma surgery. In: Kanzaki J, ed. Acoustic Neuroma: Consensus on Systems for Reporting Results. Keio University International Symposia for Life Sciences and Medicine. Vol 10. Tokyo-New York: Springer; 2003:101-105.

15. Dugar J, Nikolopoulos TP, O'Donoghue GM. Hearing preservation in acoustic neuroma surgery: The impact of different patient selection criteria. Laryngoscope. 2002;112(11):2051-2053.

16. Lassaletta L, Fontes L, Melcon E, Sarria MJ, Gavilan J. Hearing preservation with the retrosigmoid approach for vestibular schwannoma: Myth or reality? Otolaryngol Head Neck Surg. 2003;129(4):397-401.

17. Ferber-Viart C, Laoust L, Boulud B, Duclaux R, Dubreuil C. Acuteness of preoperative factors to predict hearing preservation in acoustic neuroma surgery. Laryngoscope. 2000;110(1):145-150.

18. Rohit, Piccirillo E, Jain Y, Augurio A, Sanna M. Preoperative predictive factors for hearing preservation in vestibular schwannoma surgery. Ann Otol Rhinol Laryngol. 2006;115(1):41-46.

19. Slattery WHIII, Brackmann DE, Hitselberger W. Middle fossa approach for hearing preservation with acoustic neuromas. Otol Neurotol. 1997; 18(5):596-601.

20. Brackmann DE, Owens RM, Friedman RA, et al. Prognostic factors for hearing preservation in vestibular schwannoma surgery. Otol Neurotol. 2000;21(3):417-424.

21. Rastogi $P$, Cacace AT, Lovely TJ. Factors influencing hearing preservation in acoustic tumor surgery. Skull Base Surg. 1995;5(3):137-142. 
22. Glasscock ME III, Hays JW, Minor LB, Haynes DS, Carrasco VN. Preservation of hearing in surgery for acoustic neuromas. $J$ Neurosurg. 1993;78(6):864-870.

23. Dornhoffer JL, Helms J, Hoehmann DH. Hearing preservation in acoustic tumor surgery: Results and prognostic factors. Laryngoscope. 1995;105(2):184-187.

24. Matthies C, Samii M. Management of vestibular schwannomas (acoustic neuromas): The value of neurophysiology for evaluation and prediction of auditory function in 420 cases. Neurosurgery. 1997; 40(5):919-930.
25. Browning S, Mohr G, Dufour JJ, et al. Hearing preservation in acoustic neuroma surgery. J Otolaryngol. 2001;30(5):307-315.

26. Kanzaki J, Ogawa K, Inoue Y, Shiobara R. Hearing preservation surgery in acoustic neuroma patients with normal hearing. Skull Base Surg. 1997;7(3):109-113.

27. Vincent C, Bonne N-X, Guérin C, et al. Middle fossa approach for resection of vestibular schwannoma: Impact of cochlear fossa extension and auditory monitoring on hearing preservation. Otol Neurotol. 2012;33(5):849-852.

28. Berrettini S, Ravecca F, Sellari-Franceschini S, Bruschini P, Casani A Padolecchia R. Acoustic neuroma: Correlations between morphology and otoneurological manifestations. J Neurol Sci. 1996;144(1):24-33. 